\title{
THE ORDER OF NORMAL APPROXIMATION FOR SIGNED LINEAR RANK STATISTICS*
}

\section{L. PURI ${ }^{1}$ AND T.-J. WU}

Summary. The rate of convergence of the cdf (cumulative distribution function) of the signed linear rank statistics to the normal one is investigated. Under suitable assumptions, it is shown that the convergence rate is of order $O\left(N^{-1 / 2+\delta}\right)$ for any $\delta>0$.

1. Introduction. Let $Y_{N i}, 1 \leqq i \leqq N, N \geqq 1$, be independent random variables distributed according to the cdf's $F_{N i}(y)=F\left(y-\Delta d_{N i}\right), 1 \leqq i \leqq N, N \geqq 1$, where $\Delta$ is an unknown real parameter, and $d_{N i}, 1 \leqq i \leqq N, N \geqq 1$, are known real constants. Consider the signed linear rank statistics

$$
S_{\Delta N}=\sum_{i=1}^{N} c_{N i} \varphi\left(\frac{R_{N i}^{\Delta}}{N+1}\right) \operatorname{sgn}\left(Y_{N i}\right)
$$

where $R_{N i}^{\Delta}$ is the rank of $\left|Y_{N i}\right|$ among $\left|Y_{N 1}\right|, \cdots,\left|Y_{N N}\right|, c_{N i}$ 's are known real constants, sgn $(y)=1$ or -1 according as $y \geqq 0$ or $<0$, and $\varphi$ is a score generating function defined over $(0,1)$. Under suitable assumptions on the $c$ 's and the score generating function $\varphi$, the asymptotic normality of $S_{\Delta N}$ has been established by Hušková [3]. However, the problem of determining the rate of convergence of the cdf of $S_{\Delta N}$ to the limiting normal one has not been investigated so far. The purpose of this paper is to study this problem. It is shown that the rate of convergence is of order $O\left(N^{-1 / 2+\delta}\right)$ for any $\delta>0$ for the case $\Delta=0$ as well as $\Delta \neq 0$. For the corresponding study of the similar problem in the case of (unsigned) linear rank statistics of the form

$$
S_{N}=\sum_{i=1}^{N} c_{N i} \varphi\left(\frac{R_{N i}}{N+1}\right)
$$

where $R_{N i}$ is the rank of $Y_{N i}$ among $Y_{N 1}, \cdots, Y_{N N}$, the reader is referred to Jurečková and Puri [5], Bergström and Puri [1], and Hušková [4], among others. Our methods are mainly the adaptations of the ideas of Jurečková and Puri [5], Bergström and Puri [1] and Hušková [4]. Throughout the paper we make the following assumptions:

A1) $F(x)$ is continuous and symmetric about zero,

A2) $\sum_{i=1}^{N} c_{N i}^{2}=1, \max _{1 \leqq i \leqq N}\left|c_{N i}\right|=O\left(N^{-1 / 2} \sqrt{\log N}\right)$,

A3) $\varphi$ is not identically zero and satisfies the Lipschitz condition of order one on $(0,1)$, that is, there exists a constant $C$ such that

$$
|\varphi(u)-\varphi(v)| \leqq C|u-v| \text { for all } u, v \in(0,1) .
$$

Remark. Unlike in earlier papers referred to above where the derivatives of different orders on $\varphi$ are assumed, here the Lipschitz condition on $\varphi$ is the only assumption we are making.

For simplicity of notation, we shall suppress the subscript $N$ in $c_{N i}, d_{N i}, R_{N i}^{\Delta}$ etc., whenever this causes no confusion.

2. Rate of convergence for $\Delta=0$. Here we assume that $\Delta=0$, so that $Y_{N i}, \cdots, Y_{N N}, N \geqq 1$, are i.i.d. random variables distributed according to a continuous and symmetric $\operatorname{cdf} F(y)$. We shall need the following theorem (cf. Petrov [7, Chap. V, Thm. 6]).

THEOREM 2.1. Let $V_{1}, \cdots, V_{N}$ be independent random variables with $\mathbf{E} V_{j}=0, \mathbf{E}\left|V_{j}\right|^{2+\delta}<\infty$ for some positive $\delta \leqq 1(j=1, \cdots, N)$. Then

$$
\sup _{x \in(-\infty, \infty)}\left|G_{N}(x)-\Phi(x)\right| \leqq A B_{N}^{-1-\delta / 2} \sum_{j=1}^{N} \mathbf{E}\left|V_{j}\right|^{2+\delta},
$$

where $A>0$ is a constant independent of $N, B_{N}=\sum_{j=1}^{N} \mathrm{D} V_{j}, G_{N}(x)=\mathbf{P}\left[B_{N}^{-1 / 2} \sum_{j=1}^{N} V_{j} \leqq x\right]$, and $\Phi(x)$ is the standard cumulative normal distribution function.

\footnotetext{
* Received by the editors July 13, 1982; in revised form May 20, 1985.

${ }^{1}$ Work supported by the Office of Naval Research under contract No. N00014-77-C-0659. Reproduction in whole or part is permitted for any purpose of the United States Government.

AMS 1970 subject classifications. Primary 62E20, Secondary 60F99.
} 
Now let us denote

$$
T_{\Delta N}=\sum_{i=1}^{N} c_{N i} \varphi\left(F^{*}\left(\left|Y_{N i}\right|\right)\right) \operatorname{sgn}\left(Y_{N i}\right), \quad F^{*}(x)=P_{0}\left[\left|Y_{11}\right| \leqq x\right],
$$

where $P_{0}$ means that the probability is computed under $\Delta=0$, and note that $T_{\Delta N}$ is a sum of independent random variables. We shall use $T_{\Delta N}$ to approximate $S_{\Delta N}$.

LEMMA 2.1. Under assumptions A1-A3, we have

$$
\sup _{x \in(-\infty, \infty)}\left|G_{0 N}(x)-\Phi(x)\right|=O\left(N^{-1 / 2} \sqrt{\log ^{3} N}\right),
$$

where $G_{0 N}$ is the cdf of $T_{0 N} /\|\varphi\|_{2}$ and $T_{0 N}$ is given by (2.1).

Proof. Since $\mathrm{E}\left[c_{i} \varphi\left(F^{*}\left(\left|Y_{i}\right|\right) \operatorname{sgn}\left(Y_{i}\right)\right]=0, \quad\right.$ and $\quad \mathbf{E}\left|c_{i} \varphi\left(F^{*}\left(\left|Y_{i}\right|\right)\right) \operatorname{sgn}\left(Y_{i}\right)\right|^{3}=$ $\left|c_{i}\right|^{3} \int_{0}^{1}\left|\varphi^{3}(u)\right| d u<\infty$ for $1 \leqq i \leqq N$, it follows from Theorem 2.1 that

$$
\sup _{x \in(-\infty, \infty)}\left|G_{0 N}(x)-\Phi(x)\right| \leqq A B_{N}^{-3 / 2} \sum_{i=1}^{N}\left|c_{i}\right|^{3} \int_{0}^{1}\left|\varphi^{3}(u)\right| d u,
$$

where $A>0$ is a constant independent of $N$, and $B_{N}=\sum_{i=1}^{N} c_{i}^{2} \int_{0}^{1} \varphi^{2}(u) d u$. The proof now follows from $A 2, A 3$ and (2.2).

LEMMA 2.2. Under assumptions A1-A3,

$$
\mathrm{E}\left(S_{0 N}-T_{0 N}\right)^{2 K}=O\left[N^{-K}(\log N)^{K}\right]
$$

for any fixed positive integer $K$, where $S_{0 N}$ and $T_{0 N}$ are given by (1.1) and (2.1) respectively.

Proof. Put $Z_{i}=\left[\varphi\left(R_{i}^{0} /(N+1)\right)-\varphi\left(F^{*}\left(\left|Y_{i}\right|\right)\right)\right] \operatorname{sgn}\left(Y_{i}\right)$. Then, for arbitrary nonnegative integers $p_{1}, p_{2}, \cdots, p_{N}$ satisfying $\sum_{i=1}^{N} p_{i}=2 K$ we have

$$
\mathbf{E}\left(\prod_{i=1}^{N} Z_{i}^{p_{i}}\right)=0
$$

if at least one of the $p_{i}$ 's is an odd number. Then using (2.3) we obtain

$$
\begin{aligned}
\mathbf{E}\left[\left(S_{0 N}-T_{0 N}\right)\right]^{2 K} & =\mathbf{E}\left[\left(\sum_{i=1}^{N} c_{i} Z_{i}\right)^{2 K}\right] \\
& =\sum_{\substack{l_{1}=0 \\
l_{1}+l_{2}+\cdots+l_{N}=K}}^{K} \sum_{\substack{l_{N}=0 \\
l_{2}}}^{K} C_{2 l_{N}}^{2 K} c_{1}^{2 l_{1}} c_{2}^{2 l_{2}} \cdots c_{N}^{2 l_{N}} \mathbf{E}\left(\prod_{i=1}^{N} Z_{i}^{2 l_{i}}\right) \\
& =\sum_{m=1}^{K}\left[\sum_{i_{1}=1}^{N} \sum_{\substack{i_{2}=1 \\
i_{1}<i_{2}<\cdots<i_{m}}}^{N} \cdots \sum_{i_{m}=1}^{N}\right. \\
& \quad\left[\sum_{\substack{l_{1}=1 \\
l_{1}+l_{2}+\cdots+l_{m}=K}}^{K} \sum_{l_{2}=1}^{K} \cdots \sum_{l_{m}=1}^{K} C_{21_{m}}^{2 K} c_{i_{1}}^{2 l_{1}} \cdots c_{i_{m}}^{2 l_{m}} \mathbf{E}\left(Z_{i_{1}}^{2 l_{1}} Z_{i_{2}}^{2 l_{2}} \cdots Z_{i_{m}}^{2 l_{m}}\right]\right],
\end{aligned}
$$

where

$$
\mathbf{l}_{j}=\left(l_{1}, l_{2}, \cdots, l_{j}\right) \quad \text { and } \quad C_{2 l_{j}}^{2 K}=\frac{(2 K) !}{\left(2 l_{1}\right) !\left(2 l_{2}\right) ! \cdots\left(2 l_{j}\right) !} \quad \text { for } j=N \text { or } m \text {. }
$$

Now, by the generalized Cauchy-Schwarz inequality and A3, we have for any $i_{1}<i_{2}<\cdots<i_{m}, m$; $l_{j}=1, \cdots, K ; j=1, \cdots, m, \sum_{j=1}^{m} l_{j}=K$, that

$$
\begin{aligned}
\mathbf{E}\left(Z_{i_{1}}^{2 l_{1}} Z_{i_{2}}^{2 l_{2}} \cdots Z_{i_{m}}^{2 l_{m}}\right) & \leqq\left(\prod_{j=1}^{m} \mathbf{E}\left(Z_{j}^{2 m l_{j}}\right)\right)^{1 / m} \\
& =\left\{\prod_{j=1}^{m} \mathbf{E}\left[\varphi\left(R_{1}^{0} /(N+1)\right)-\varphi\left(F^{*}\left(\left|Y_{1}\right|\right)\right)\right]^{2 m l_{j}}\right\}^{1 / m} \\
& \leqq C^{2 K}\left\{\prod_{j=1}^{m} \mathbf{E}\left(\frac{R_{1}^{0}}{N+1}-F^{*}\left(\left|Y_{1}\right|\right)\right)^{2 m l_{j}}\right\}^{1 / m} \leqq M_{k} N^{-K},
\end{aligned}
$$


where $M_{K}$ is a constant depending only on $K$. To obtain the last inequality in (2.5) we have used the well-known fact (see e.g. Kallenberg [6, p. 406]) that

$$
\mathrm{E}\left(\frac{R_{1}^{0}}{N+1}-F^{*}\left(\left|Y_{1}\right|\right)\right)^{2 p}=O\left(N^{-p}\right)
$$

for any positive integer $p$. Also, in view of A2, there exists a constant $M_{K}^{\prime}$ (depending only on $K)$ such that

$$
\left|c_{i_{1}}^{2 l_{1}} c_{i_{2}}^{2 l_{2}} \cdots c_{i_{m}}^{2 l_{m}}\right| \leqq M_{K}^{\prime} N^{-K}(\log N)^{K}
$$

for any $i_{1}<\cdots<i_{m}, m=1, \cdots, K$, and any $l_{j}=1, \cdots, K ; j=1, \cdots, m, \sum_{j=1}^{m} l_{j}=K$. The proof now follows from (2.4), (2.5) and (2.6).

The main theorem of this section is the following.

THEOREM 2.2. Under assumptions A1-A3, we have for any $\delta>0$ that

$$
\sup _{x \in(-\infty, \infty)}\left|E_{0 N}(x)-\varphi(x)\right|=O\left(N^{-1 / 2+\delta}\right),
$$

where $F_{0 N}$ is the cdf of $S_{0 N} / \varphi \|_{2}$.

Proof. For any $\varepsilon>0$ and any $N$, we have

$$
\mathbf{P}\left[\|\Phi\|_{2}^{-1} S_{0 N} \leqq x\right] \leqq \mathbf{P}\left[\|\Phi\|_{2}^{-1} T_{0 N} \leqq x+\varepsilon\right]+\mathbf{P}\left[\|\varphi\|_{2}^{-1}\left|S_{0 N}-T_{0 N}\right| \geqq \varepsilon\right]
$$

and

$$
\mathbf{P}\left[\|\Phi\|_{2}^{-1} S_{0 N} \leqq x\right] \geqq \mathbf{P}\left[\|\Phi\|_{2}^{-1} T_{0 N} \leqq x-\varepsilon\right]-\mathbf{P}\left[\|\Phi\|_{2}^{-1}\left|S_{0 N}-T_{0 N}\right| \geqq \varepsilon\right] .
$$

It then follows from (2.7), (2.8), Lemma 2.1, Lemma 2.2 and Markov's inequality that for any $\delta>0$

$$
\sup _{x \in(-\infty, \infty)}\left|F_{0 N}(x)-\Phi(x)\right| \leqq O\left(N^{-1 / 2} \sqrt{\log ^{3} N}\right)+O(\varepsilon)+\frac{O\left(N^{-K}(\log N)^{K}\right)}{\left(\varepsilon\|\Phi\|_{2}\right)^{2 K}}
$$

holds for any $\varepsilon>0$ and any $K \geqq 1$. Now, pick $K$ large enough such that $(2 K+1)^{-1}<2 \delta$ and put $\varepsilon=N^{-1 / 2+1 /(2(2 K+1))}$. Then using (2.9), the proof follows immediately.

3. Rate of convergence under near alternatives. In this section, we assume $\Delta \neq 0$ so that $Y_{N 1}, \cdots, Y_{N N}, N \geqq 1$, are independently distributed with cdf's $F\left(y-\Delta d_{N_{1}}\right), \cdots, F\left(y-\Delta d_{N N}\right)$, $N \geqq 1$, respectively. Furthermore, besides the assumptions A1-A3, we assume:

B1) $F$ is absolutely continuous, $f(x)=F^{\prime}(x)$ is absolutely continuous, and there exists a constant $\theta_{0}>0$ such that

$$
\sup _{\theta \in\left[-\theta_{0}, \theta_{0}\right]} \int_{-\infty}^{\infty} \frac{\left[f^{\prime}(x-\theta)\right]^{2}}{f(x)} d x<\infty
$$

and

$$
\sum_{i=1}^{N} d_{N i}^{2}=1, \quad \sum_{i=1}^{N} d_{N i}=0, N=2,3, \cdots, \max _{1 \leqq i \leqq N}\left|d_{N i}\right|=O\left(N^{-1 / 2} \sqrt{\log N}\right) .
$$

In what follows $\mathbf{E}_{0}$ and $\mathbf{E}_{\Delta}$ will denote the expectations computed under $\Delta=0$ and $\Delta \neq 0$ respectively. Furthermore, $P_{\Delta r}$ will denote the restriction of $P_{\Delta}$ to the set $D_{N}=\left\{\prod_{i=1}^{N} f\left(Y_{N i}\right) \neq 0\right\}$ so that for any measurable function $h$ of $\left(Y_{1}, Y_{2}, \cdots, Y_{N}\right)$

$$
\begin{aligned}
\mathbf{E}_{\Delta r} h\left(Y_{1}, Y_{2}, \cdots, Y_{N}\right)= & \int_{\left\{\prod_{i=1}^{N} f\left(y_{i}\right) \neq 0\right\}} h\left(y_{1}, y_{2}, \cdots, y_{N}\right) \\
& \cdot \prod_{i=1}^{N} f\left(y_{i}-\Delta d_{i}\right) d y_{1} d y_{2} \cdots d y_{N},
\end{aligned}
$$

i.e., $\mathbf{E}_{\Delta r} h\left(Y_{1}, Y_{2}, \cdots, Y_{N}\right)=\mathbf{E}_{\Delta}\left\{h\left(Y_{1}, Y_{2}, \cdots, Y_{n}\right) I_{D_{N}}\left(Y_{1}, Y_{2}, \cdots, Y_{N}\right)\right\}$, where $I$ denotes the indicator function.

LEMMA 3.1. Under assumptions $\mathrm{A} 1, \mathrm{~B} 1$ and $\mathrm{B} 2$,

$$
P_{\Delta}\left(D_{N}^{c}\right)=O\left(N^{-1 / 2} \sqrt{\log ^{3} N}\right)
$$

holds for any $\Delta \neq 0$, where $D_{N}^{c}$ is the complement of $D_{N}$.

Remark. Our assumption $B 1$ is equivalent to assumption $V$ in Hušková [4]. In fact, Hušková's assumption V implies our assumption B1 and her Lemma 3.5 implies our Lemma 3.1. 
Proof. By Lemma 3.5 of Hušková [4] and B2, there exists a constant $C_{1}$ (independent of $N)$ and a positive integer $N_{\Delta}$ such that for all $N \geqq N_{\Delta}$ we have

$$
P_{\Delta}\left[\prod_{i=1}^{N} f\left(Y_{N i}\right)=0\right] \leqq C_{1}|\Delta|^{3} \sum_{i=1}^{N}\left|d_{i}\right|^{3}=O\left(N^{-1 / 2} \sqrt{\log ^{3} N}\right) .
$$

The proof follows.

LEMMA 3.2. Under assumptions $\mathrm{A} 1$ to $\mathrm{A} 3, \mathrm{~B} 1$ and $\mathrm{B} 2$, it holds true for any positive integer $K$ and any $\Delta \neq 0$ that

$$
\left\{\mathbf{E}_{\Delta}\left[\left(S_{\Delta N}-T_{\Delta N}\right) I_{D_{N}^{c}}\left(Y_{1}, \cdots, Y_{N}\right)\right]\right\}^{2 K}=O\left[\left(N^{-K}(\log N)^{3 K}\right)^{1-1 /(2 p)}(\log N)^{2 K}\right]
$$

for all $p=1,2, \cdots$.

Proof. By the Hölder inequality, the left-hand side of (3.2) is bounded by

$$
\left[\mathbf{E}_{\Delta}\left(S_{\Delta N}-T_{\Delta N}\right)^{2 p}\right]^{K / p}\left[P_{\Delta}\left(D_{N}^{c}\right)\right]^{2 K(1-1 /(2 p))}
$$

for any $p=1,2, \cdots$. Thus the assertion (3.2) will be proved in view of (3.3) and Lemma 3.1, if we can show that $\mathbf{E}_{\Delta}\left(S_{\Delta N}-T_{\Delta N}\right)^{2 p}=O\left[(\log N)^{2 p}\right]$ for any $p=1,2, \cdots$. Now, put

$$
Z_{i \Delta}=\left[\varphi\left(\frac{R_{i}^{\Delta}}{N+1}\right)-\varphi\left(F^{*}\left(\left|Y_{i}\right|\right)\right)\right] \operatorname{sgn}\left(Y_{i}\right)
$$

Then from (1.1), (2.1) and (2.4) we have

$$
\begin{aligned}
\mathbf{E}_{\Delta}\left(S_{\Delta N}-T_{\Delta N}\right)^{2 p} & =\mathbf{E}_{\Delta}\left[\left(\sum_{i=1}^{N} c_{i} Z_{i \Delta}\right)^{2 p}\right] \\
& =\sum_{m=1}^{2 p}\left[\sum_{i_{1}=1}^{N} \cdots \sum_{i_{1}<\cdots<i_{m}=1}^{N}\left[\sum_{l_{1}=1}^{2 p} \cdots \sum_{l_{1}=1}^{2 p} C_{l_{m}+\cdots+l_{m}=2 p}^{2 p} c_{i_{1}^{1}}^{l_{1}} \cdots c_{i_{m}}^{l_{m}} \mathbf{E}_{\Delta}\left(Z_{i_{1} \Delta}^{l_{1}} \cdots Z_{i_{m} \Delta}^{l_{m}}\right)\right]\right],
\end{aligned}
$$

where

$$
C_{\mathbf{1}_{m}}^{2 p}=\frac{(2 p) !}{\left(l_{1} !\right) \cdots\left(l_{m} !\right)^{\circ}}
$$

We now consider the expectation $\mathbf{E}_{\Delta} Z_{i \Delta}^{2 q}$ for any $q=1,2, \cdots$ and any $i=1, \cdots, N$.

From $\mathbf{A} 3$ it follows that

$$
\begin{aligned}
\mathbf{E}_{\Delta} Z_{i \Delta}^{2 q} & \leqq C^{2 q} \mathbf{E}_{\Delta}\left(\frac{R_{i}^{\Delta}}{N+1}-F^{*}\left(\left|Y_{i}\right|\right)\right)^{2 q} \\
& \leqq 2^{2 q-1} C^{2 q}\left[\mathbf{E}_{\Delta}\left(\frac{R_{i}^{\Delta}}{N+1}-V_{i i \Delta}\right)^{2 q}+\mathbf{E}_{\Delta}\left(V_{i i \Delta}-F^{*}\left(\left|Y_{i}\right|\right)\right)^{2 q}\right],
\end{aligned}
$$

where $V_{i i \Delta}=\mathbf{E}_{\Delta}\left(R_{i}^{\Delta} /(N+1) \mid Y_{i}\right)$. By Lemma 2.1 (after slight and trivial modification) of Bergström and Puri [1] we obtain

$$
\mathbf{E}_{\Delta}\left(\frac{R_{i}^{\Delta}}{N+1}-V_{i i \Delta}\right)^{2 q}=O\left(N^{-q}\right), \quad q=1,2, \cdots,
$$

uniformly in $i=1,2, \cdots, N$. Using the Mean Value Theorem and B1, we can easily show that

$$
\mathbf{E}_{\Delta}\left(V_{i i \Delta}-F^{*}\left(\left|Y_{i}\right|\right)\right)^{2 q}=O\left(\max _{i \leqq j \leqq N}\left|d_{j}\right|^{2 q}\right), \quad q=1,2, \cdots,
$$

uniformly in $i=1,2, \cdots, N$. It now follows from B2, (3.5), (3.6) and (3.7) that

$$
\mathbf{E}_{\Delta} Z_{i \Delta}^{2 q}=O\left[N^{-q}(\log N)^{q}\right], \quad q=1,2, \cdots,
$$

holds uniformly in $i=1, \cdots, N$. In view of (3.4) and (3.8), it follows, by using arguments analogous to those in the proof of Lemma 2.2, that

$$
\mathbf{E}_{\Delta}\left(S_{\Delta N}-T_{\Delta N}\right)^{2 p}=O\left[(\log N)^{2 p}\right], \quad p=1,2, \cdots .
$$

The proof follows.

LEMMA 3.3. Under assumptions $\mathrm{A} 1-\mathrm{A} 3, \mathrm{~B} 1$ and $\mathrm{B} 2$, it holds for any $K=1,2, \cdots$ and any $\Delta \neq 0$ that

$$
\mathbf{E}_{\Delta r}\left[\left(S_{\Delta N}-\mathbf{E}_{\Delta} S_{\Delta N}\right)-\left(T_{\Delta N}-\mathbf{E}_{\Delta} T_{\Delta N}\right)\right]^{2 K}=O\left[N^{-K(1-1 /(2 p))}(\log N)^{q_{K p}}\right]
$$

for all $p=1,2, \cdots$, where $q_{K p}=(5-3 /(2 p)) K$. 
Proof. The left-hand side of (3.10) is bounded by

$$
2^{2 K-1} \mathbf{E}_{\Delta r}\left(S_{\Delta N}-T_{\Delta N}\right)^{2 K}+2^{2 K-1}\left[E_{\Delta}\left(S_{\Delta N}-T_{\Delta N}\right)\right]^{2} K \text {. }
$$

In view of Lemma 3.6 of Hušková [4], there exist a constant $C_{2}$ (independent of $N$ ) and a positive integer $N_{\Delta}^{\prime}$ such that for all $N \geqq N_{\Delta}^{\prime}$

$$
\mathbf{E}_{\Delta r}\left(S_{\Delta N}-T_{\Delta N}\right)^{2 K} \leqq C_{2}\left\{\mathbf{E}_{0}\left(S_{0 N}-T_{0 N}\right)^{4 K}\right\}^{1 / 2}=O\left[N^{-K}(\log N)^{K}\right],
$$

where the last equality is by Lemma 2.2 . Next, we have

$$
\begin{aligned}
& {\left[\mathbf{E}_{\Delta}\left(S_{\Delta N}-T_{\Delta N}\right)\right]^{2 K}=\left\{\mathbf{E}_{\Delta r}\left(S_{\Delta N}-T_{\Delta N}\right)+\mathbf{E}_{\Delta}\left[\left(S_{\Delta N}-T_{\Delta N}\right) I_{D_{N}^{c}}\left(Y_{1}, \cdots, Y_{N}\right)\right]\right\}^{2 K}} \\
& \quad \leqq 2^{2 K-1}\left[\mathbf{E}_{\Delta r}\left(S_{\Delta N}-T_{\Delta N}\right)\right]^{2 K}+2^{2 K-1}\left\{\mathbf{E}_{\Delta}\left[\left(S_{\Delta N}-T_{\Delta N}\right) I_{D_{N}^{c}}\left(Y_{1}, \cdots, Y_{N}\right)\right]\right\}^{2 K} .
\end{aligned}
$$

Using (3.1) and Jensen's inequality, it follows that

$$
\left[\mathbf{E}_{\Delta r}\left(S_{\Delta N}-T_{\Delta N}\right)\right]^{2 K} \leqq \mathbf{E}_{\Delta r}\left(S_{\Delta N}-T_{\Delta N}\right)^{2 K}
$$

The proof now follows from (3.11)-(3.14) and Lemma 3.2.

The following lemma is an immediate consequence of the previous lemmas and the Markov inequality.

LEMMA 3.4. Under assumptions $\mathrm{A} 1-\mathrm{A} 3, \mathrm{~B} 1$ and $\mathrm{B} 2$, it holds true for any $\Delta \neq 0$ and $\varepsilon>0$ that

$$
\begin{aligned}
& P_{\Delta}\left[\left|\left(S_{\Delta N}-\mathbf{E}_{\Delta} S_{\Delta N}\right)-\left(T_{\Delta N}-\mathbf{E}_{\Delta} T_{\Delta N}\right)\right|\|\varphi\|_{2}^{-1}>\varepsilon\right] \\
& \quad \leqq M_{K P}\left[N^{-1 / 2} \sqrt{\log ^{3} N}+\left(\|\varphi\|_{2} \varepsilon\right)^{-2 K} N^{-K(1-1 /(2 p))}(\log N)^{q_{K p}}\right]
\end{aligned}
$$

for all $K, p=1,2, \cdots$ and for all $N \geqq N_{\Delta K p}$ where $N_{\Delta K p}$ is a positive integer, $M_{K p}$ is a constant independent of $N$ and $q_{K p}=(5-3 /(2 p)) K$.

Proof. For any $\varepsilon>0$, the left-hand of (3.15) is bounded by

$$
P_{\Delta}\left(D_{N}^{c}\right)+P_{\Delta r}\left[\left|\left(S_{\Delta N}-\mathbf{E}_{\Delta} S_{\Delta N}\right)-\left(T_{\Delta N}-\mathbf{E}_{\Delta} T_{\Delta N}\right)\right|\|\varphi\|_{2}^{-1}>\varepsilon\right] .
$$

(Recall from (3.1) that $P_{\Delta r}(A)=P_{\Delta}\left(A \cap D_{N}\right)$ for any measurable set $A$.)

The first term in (3.16) is of order $O\left(N^{-1 / 2} \sqrt{\log ^{3} N}\right)$ as can be seen from Lemma 3.1. The second term in (3.16), by Markov's inequality, is bounded by

$$
\mathbf{E}_{\Delta r}\left[\left(S_{\Delta N}-\mathbf{E}_{\Delta} S_{\Delta N}\right)-\left(T_{\Delta N}-\mathbf{E}_{\Delta} T_{\Delta N}\right)\right]^{2 K}\left(\|\varphi\|_{2} \varepsilon\right)^{-2 K} \text { for any } K=1,2, \cdots .
$$

The lemma now follows from Lemma 3.3.

THEOREM 3.1. Under assumptions $\mathrm{A} 1-\mathrm{A} 3, \mathrm{~B} 1$ and $\mathrm{B} 2$, it holds true that

$$
\sup _{x \in(-\infty, \infty)}\left|G_{\Delta N}(x)-\Phi(x)\right|=O\left(N^{-1 / 2} \sqrt{\log ^{3} N}\right),
$$

where $G_{\Delta N}$ is the cdf of $\left(T_{\Delta N}-\mathbf{E}_{\Delta} T_{\Delta N}\right)\|\varphi\|_{2}^{-1}$ and $T_{\Delta N}$ is given by (2.1).

Proof. Let us denote $W_{i \Delta}=\varphi\left(F^{*}\left(\left|Y_{i}\right|\right) \operatorname{sgn}\left(Y_{i}\right)\right.$. Then using (2.1), we have $T_{\Delta N}-\mathbf{E}_{\Delta} T_{\Delta N}=$ $\sum_{i=1}^{N} c_{i}\left(W_{i \Delta}-E_{\Delta} W_{i \Delta}\right)$ which is the sum of independent random variables, and by $\mathrm{A} 2$ we have

$$
\begin{aligned}
\left|\mathbf{D}_{\Delta} T_{\Delta N}-\|\varphi\|_{2}^{2}\right| & =\left|\sum_{i=1}^{N} c_{i}^{2}\left[\mathbf{E}_{\Delta} W_{i \Delta}^{2}-\|\varphi\|_{2}^{2}-\left(\mathbf{E}_{\Delta} W_{i \Delta}\right)^{2}\right]\right| \\
& \leqq \max _{1 \leqq i \leqq N}\left|\mathbf{E}_{\Delta} W_{i \Delta}^{2}-\|\varphi\|_{2}^{2}\right|+\max _{1 \leqq i \leqq N}\left|\mathbf{E}_{\Delta} W_{i \Delta}\right|^{2} .
\end{aligned}
$$

Now, using A1, A3, B1 and the Mean Value Theorem we obtain

$$
\begin{aligned}
\max _{1 \leqq i \leqq N}\left|\mathrm{E}_{\Delta} E_{i \Delta}^{2}-\|\varphi\|_{2}^{2}\right| \leqq & \max _{1 \leqq i \leqq N} \int_{-\infty}^{\infty}\left|\varphi^{2}\left(F^{*}\left(\left|x+\Delta d_{i}\right|\right)\right)-\varphi^{2}\left(F^{*}(|x|)\right)\right| d F(x) \\
\leqq & 2\|\varphi\|_{\infty} C \max _{1 \leqq i \leqq N} \int_{-\infty}^{\infty}\left|F^{*}\left(\left|x+\Delta d_{i}\right|\right)-F^{*}(|x|)\right| d F(x) \\
\leqq & 2\|\varphi\|_{\infty} C \int_{-\infty}^{\infty}\left[F^{*}\left(|x|+\max _{1 \leqq i \leqq N}\left|\Delta d_{i}\right|\right)\right. \\
& \left.-F^{*}\left(|x|-\max _{1 \leqq i \leqq N}\left|\Delta d_{i}\right|\right)\right] d F(x) \\
= & O\left(\max _{1 \leqq i \leqq N}\left|d_{i}\right|\right)=O\left(N^{-1 / 2} \sqrt{\log N}\right)
\end{aligned}
$$


and similarly

$$
\begin{aligned}
\max _{1 \leqq i \leqq N}\left|\mathbf{E}_{\Delta} W_{i \Delta}\right|= & \max _{1 \leqq i \leqq N}\left|\mathbf{E}_{\Delta} W_{i \Delta}-\mathbf{E}_{0} W_{i 0}\right| \\
\leqq & \max _{1 \leqq i \leqq N} \int_{M_{i \Delta}}^{\infty}\left|\varphi\left(F^{*}\left(x+\Delta d_{i}\right)\right)-\varphi\left(F^{*}(x)\right)\right| d F(x) \\
& +\max _{1 \leqq i \leqq N} \int_{-\infty}^{m_{i \Delta}}\left|\varphi\left(F^{*}(-x)\right)-\varphi\left(F^{*}\left(-x-\Delta d_{i}\right)\right)\right| d F(x) \\
& +\max _{1 \leqq i \leqq N} \int_{m_{i \Delta}}^{M_{i \Delta}} \mid \varphi\left(F^{*}\left(\left|x+\Delta d_{i}\right|\right)\right) \operatorname{sgn}\left(x+\Delta d_{i}\right) \\
& -\varphi\left(F^{*}(|x|)\right) \operatorname{sgn}(x) \mid d F(x),
\end{aligned}
$$

where $M_{i \Delta}=\max \left(0,-\Delta d_{i}\right)$ and $m_{i \Delta}=\min \left(0,-\Delta d_{i}\right)$. The third integral in (3.19) is of order $O\left(\max _{1 \leqq i \leqq N}\left|d_{i}\right|\right)$ since $\|\Phi\|_{\infty}<\infty$ and $F\left(M_{i \Delta}\right)-F\left(m_{i \Delta}\right)=O\left(\max _{1 \leqq i \leqq N}\left|d_{i}\right|\right)$. The first and second integrals are also of order $O\left(\max _{1 \leqq i \leqq N}\left|d_{i}\right|\right)$, which can be proved as in deriving (3.18). It then follows from B2, (3.17), (3.18) and (3.19) that

$$
\left|\mathbf{D}_{\Delta} T_{\Delta N}-\|\varphi\|_{2}^{2}\right|=O\left(N^{-1 / 2} \sqrt{\log N}\right) .
$$

But A3 implies that $\|\varphi\|_{2}>0$. Thus there exists a positive integer $N_{\Delta}^{\prime \prime}$ such that $\frac{1}{2}\|\varphi\|_{2}^{2}<\mathbf{D}_{\Delta} T_{\Delta N}<$ $\frac{3}{2}\|\varphi\|_{2}^{2}$ for all $N \geqq N_{\Delta}^{\prime \prime}$. Consequently we have for all $N \geqq N_{\Delta}^{\prime \prime}$,

$$
\begin{aligned}
\left|-\frac{\|\varphi\|_{2}}{\sqrt{D_{\Delta} T_{\Delta N}}}-1\right| & =\frac{\left|\left(\|\varphi\|_{2}-\sqrt{D_{\Delta} T_{\Delta N}}\right)\left(\|\Phi\|_{2}+\sqrt{D_{\Delta} T_{\Delta N}}\right)\right|}{\sqrt{D_{\Delta} T_{\Delta N}}\left(\|\varphi\|_{2}+\sqrt{D_{\Delta} T_{\Delta N}}\right)} \\
& \leqq \frac{3\|\varphi\|_{2}^{-2}}{\sqrt{2}+1}\left|\|\varphi\|_{2}^{2}-D_{\Delta} T_{\Delta N}\right|=O\left(N^{-1 / 2} \sqrt{\log N}\right) .
\end{aligned}
$$

Now let us denote the cdf of $\left(T_{\Delta N}-\mathbf{E}_{\Delta} T_{\Delta N}\right)\left(\mathbf{D}_{\Delta} T_{\Delta N}\right)^{-1 / 2}$ by $G_{\Delta N}^{*}$. Since $\mathbf{E}_{\Delta}\left|c_{i}\left(W_{i \Delta}-\mathbf{E}_{\Delta} W_{i \Delta}\right)\right|^{3}<$ $\infty, i=1, \cdots, N$, we obtain from Theorem 2.1 and (3.20) that there exists a constant $A$ (independent on $N$ ) such that for all $N \geqq N_{\Delta}^{\prime \prime}$,

$$
\begin{aligned}
\sup _{x \in(-\infty, \infty)}\left|G_{\Delta N}^{*}(x)-\varphi(x)\right| & \leqq A\left(\mathbf{D}_{\Delta} T_{\Delta N}\right)^{-3 / 2} \sum_{i=1}^{N}\left|c_{i}\right|^{3} \mathbf{E} W_{i \Delta}-\left.\mathbf{E}_{\Delta} W_{i \Delta}\right|^{3} \\
& \leqq 16 \sqrt{2} A\|\varphi\|_{2}^{-3}\|\varphi\|_{\infty}^{3} \sum_{i=1}^{N}\left|c_{i}\right|^{3} .
\end{aligned}
$$

We now consider the uniform distance between $G_{\Delta N}$ and $\Phi$. It follows from the triangle inequality and (3.22) that

$$
\begin{aligned}
\sup _{x \in(-\infty, \infty)}\left|G_{\Delta N}(x)-\Phi(x)\right|= & \sup _{x \in(-\infty, \infty)}\left|G_{\Delta N}^{*}\left(x\|\varphi\|_{2}\left(\mathbf{D}_{\Delta} T_{\Delta N}\right)^{-1 / 2}\right)-\Phi(x)\right| \\
\leqq & \sup _{x \in(-\infty, \infty)}\left|G_{\Delta N}^{*}\left(x\|\varphi\|_{2}\left(\mathbf{D}_{\Delta} T_{\Delta N}\right)^{-1 / 2}\right)-\Phi\left(x\|\varphi\|_{2}\left(\mathbf{D}_{\Delta} T_{\Delta N}\right)^{-1 / 2}\right)\right| \\
& \quad+\sup _{x \in(-\infty, \infty)}\left|\Phi\left(x\|\varphi\|_{2}\left(\mathbf{D}_{\Delta} T_{\Delta N}\right)^{-1 / 2}\right)-\Phi(x)\right| \\
\leqq & O\left(\sum_{i=1}^{N}\left|c_{i}\right|^{3}\right)+\sup _{|x| \leqq \sqrt{\log N}} \| \Phi\left(x\|\varphi\|_{2}\left(\mathbf{D}_{\Delta} T_{\Delta N}\right)^{-1 / 2}\right)-\Phi(x) \mid \\
& \quad+\sup _{|x|>\sqrt{\log N}}\left|\Phi\left(x\|\varphi\|_{2}\left(\mathbf{D}_{\Delta} T_{\Delta N}\right)^{-1}\right)-\Phi(x)\right| .
\end{aligned}
$$

On the right-hand side of the last inequality in (3.23), the second term, in view of (3.21) and the Mean Value Theorem, is bounded by

$$
\left\|\Phi^{\prime}\right\|_{\infty} \sqrt{\log N}\left\|\frac{\|\varphi\|^{2}}{\sqrt{D_{\Delta} T_{\Delta N}}}-1\right\|=O\left(N^{-1 / 2} \log N\right),
$$


and the third term, by the triangle inequality and the monotonicity of $\Phi$, is bounded by

$$
\begin{aligned}
\sup _{x>\sqrt{ } \log N}\left|1-\Phi\left(x\|\varphi\|_{2}\left(\mathbf{D}_{\Delta} T_{\Delta N}\right)^{-1 / 2}\right)\right|+\sup _{x>\sqrt{\log N}}|1-\Phi(x)| \\
=\left[1-\Phi\left(\sqrt{\log N}\|\varphi\|_{2}\left(\mathbf{D}_{\Delta} T_{\Delta N}\right)^{-1 / 2}\right)\right]+[1+\Phi(\sqrt{\log N})] \\
\left.=\left[\Phi(\sqrt{\log N})-\Phi(\sqrt{\log N})\|\varphi\|_{2}\left(\mathbf{D}_{\Delta} T_{\Delta N}\right)^{-1 / 2}\right)\right]+2[1-\Phi(\sqrt{\log N})]
\end{aligned}
$$

which is of order $O\left(N^{-1 / 2} \log N\right)$ as can be seen from (3.21) and from Lemma 2, Chapter VII of Feller [2]. The rest of the proof follows from A2, (3.23), (3.24) and (3.25).

We now prove the main result of this section.

THEOREM 3.2. Under assumptions $\mathrm{A} 1-\mathrm{A} 3, \mathrm{~B} 1$ and $\mathrm{B} 2$, it holds true for any $\delta>0$ that

$$
\sup _{x \in(-\infty, \infty)}\left|F_{\Delta N}(x)-\Phi(x)\right|=O\left(N^{-1 / 2+\delta}\right),
$$

where $F_{\Delta N}$ is the cdf of $\left(S_{\Delta N}-\mathbf{E}_{\Delta} S_{\Delta N}\right)\|\Phi\|_{2}^{-1}$.

Proof. For any $\varepsilon>0$ and any real number $x$, we have

$$
\begin{aligned}
P_{\Delta}\left[\left(S_{\Delta N}-\mathbf{E}_{\Delta} S_{\Delta N}\right)\|\varphi\|_{2}^{-1} \leqq x\right] \leqq & P_{\Delta}\left[\left(T_{\Delta N}-\mathbf{E}_{\Delta} T_{\Delta N}\right)\|\varphi\|_{2}^{-1} \leqq x+\varepsilon\right] \\
& +P_{\Delta}\left[\left(S_{\Delta N}-\mathbf{E}_{\Delta} S_{\Delta N}\right)-\left(T_{\Delta N}-\mathbf{E}_{\Delta} T_{\Delta N}\right) \mid\|\varphi\|_{2}^{-1}>\varepsilon\right]
\end{aligned}
$$

and

$$
\begin{aligned}
P_{\Delta}\left[\left(S_{\Delta N}-\mathbf{E}_{\Delta} S_{\Delta N}\right)\|\varphi\|_{2}^{-1} \leqq x\right] \geqq & P_{\Delta}\left[\left(T_{\Delta N}-\mathbf{E}_{\Delta} T_{\Delta N}\right)\|\varphi\|_{2}^{-1} \leqq x-\varepsilon\right] \\
& -P_{\Delta}\left[\left|\left(S_{\Delta N}-\mathbf{E}_{\Delta} S_{\Delta N}\right)-\left(T_{\Delta N}-\mathbf{E}_{\Delta} T_{\Delta N}\right)\right|\|\varphi\|_{2}^{-1}>\varepsilon\right] .
\end{aligned}
$$

It follows from (3.26), (3.27), Lemma 3.4 and Theorem 3.1 that for all large $N$

$$
\begin{aligned}
\sup _{x \in(-\infty, \infty)}\left|F_{\Delta N}(x)-\Phi(x)\right| \leqq & O\left(N^{-1 / 2} \sqrt{\log ^{3} N}\right)+O(\varepsilon) \\
& +\left(\|\varphi\|_{2} \varepsilon\right)^{-2 K} O\left[N^{-(1-1 /(2 p)) K}(\log N)^{(5-3 /(2 p)) K}\right]
\end{aligned}
$$

holds for any $K, p=1,2, \cdots$, and any $\varepsilon>0$. Now, pick $K$ large enough so that $(2 K+1)^{-1}<\delta$, let $p=K(2 K+1)$, and put $\varepsilon=N^{-1 / 2}+1 /(2(2 K+1))$. The theorem then follows from (3.28).

\section{REFERENCES}

[1] H. Bergström ANd M. L. Puri, Convergence and remainder terms in linear rank statistics, Ann. Statist., 4 (1977), pp. 671-680.

[2] W. Feller, An Introduction to Probability Theory and Its Applications, Vol. 2, 2nd ed., John Wiley, New York, 1971.

[3] M. Hǔsková, Asymptotic distribution of simple linear rank statistics for testing symmetry, Z. Wahrsch. Verw. Gebiete, 14 (1970), pp. 308-322.

[4] - The rate of convergence of simple linear rank statistics under hypothesis and alternatives, Ann. Statist., 5 (1977), pp. 658-670.

[5] J. JURECKOVÁ AND M. L. PURI, Order of normal approximation for rank test statistics distribution, Ann. Probab., 3 (1975), pp. 526-533.

[6] W. C. M. KALlenberG, Cramér type large deviations for simple linear rank statistics, Z. Wahrsch. Verw. Gebiete, 60 (1982), pp. 403-409.

[7] V. V. Petrov, Sums of Independent Random Variables, Springer-Verlag, New York, Berlin, Heidelberg, 1975. 
Reproduced with permission of the copyright owner. Further reproduction prohibited without permission. 